\title{
Hacia una lectura de la violencia en la formación de la subjetividad
}

\section{Scanning Violence in the Construction of Subjectivity}

\author{
Marcela Amador Calvo' \\ División de Educación para el trabajo \\ Centro de Investigación y Docencia en Educación \\ Universidad Nacional \\ Heredia, Costa Rica \\ marcelaamadorcalvo@yahoo.com.ar
}

Recibido 18 de junio de 2012 C Corregido 29 de setiembre de 2012 • Aceptado 07 de noviembre de 2012

Resumen. Este ensayo presenta un recorrido general en la construcción de la subjetividad de los sujetos; desde una perspectiva analítico vincular, plantea cómo la intrasubjetividad de cada uno es un producto del interjuego de lo intersubjetivo y transubjetivo, y cómo el estar inmerso en grupos violentos, tanto familiares como escolares, produce que la violencia forme parte de la identidad de los infantes, y los convierte, así, en futuros reproductores de tramas vinculares violentas.

Palabras claves. Violencia, identidad, infantes, familia, grupo.

Abstract. This paper provides an overview of the construction of subjectivity from an analyticalbinding perspective. It explains how the inter-subjectivity of each individual is the result of interplay between inter-subjectivity and trans-subjectivity. It also explains how violence becomes part of the children's identity when they grow up in a violent environment, at family or school, making them to reproduce the same kind of environment in the future.

Keywords. Violence, identity, children, family, group.

La violencia es un fenómeno multicausal y complejo, con múltiples aristas. Para su abordaje, es indispensable una reflexión sobre las distintas dimensiones involucradas en la problemática.

La teoría analítico vincular plantea que dicho fenómeno debe ser analizado desde el vínculo en sus aspectos intra, inter y transubjetivos. Se entiende por intrasubjetivo el mundo intrapsíquico de los sujetos, su mundo interno, lo que hace singular a cada quien. Por el área intersubjetiva, el entramado de subjetividades, producto de la configuración vincular que establecen los sujetos reales que conforman los grupos de pertenencia, familia, y otros significativos, como amigos y amigas, compañeros y compañeras escolares, etc. $Y$

Psicóloga. Máster en Estudios en Psicología Grupal. Máster en Psicología Clínica Grupal. Docente Universitaria. Psicóloga Institucional. 
URL: http://www.una.ac.cr/educare

transubjetividad refiere a aquellos significantes y significados socioculturales inconscientes presentes en el colectivo social (Krakov y Pachuk,1998).

Esta postura teórica permite una visión global de la problemática, sin poner el acento solo en lo individual, ni solo en lo social, sino en la interacción de ambas esferas. Sin embargo, para efectos de presentación, se expondrá cada uno de estas dimensiones por separado, sin perder de vista que estas operan de una manera articulada e integral.

\section{Los vínculos y la construcción de la subjetividad}

Se parte del hecho de que para existir como personas se necesita de otros individuos. Primeramente se requiere del deseo consciente o inconsciente de otros y otras para poder tener vida, y para poder mantenerla.

En la época de gestación, el bebé o la bebé se alimenta, se protege y sostiene a través de una madre que le mantiene con vida. En esta etapa hay una fusión entre este ser y la madre, por lo que aún no se le considera como un sujeto diferente.

Incluso, una vez salido del útero, el recién nacido es tan dependiente, que requiere de una matriz extrauterina que le contenga, le sostenga del desamparo e indefensión propias de su condición; una matriz que le proteja mientras va madurando, desarrollando y organizando otras áreas necesarias para sobrevivir: anatómicas, fisiológicas y psicológicas. En esta etapa, pese a ser un ser objetivamente separado, aún se considera que subjetivamente está fusionado a la madre. La madre, o quien realice la función materna, comparte con el bebé o la bebé el campo subjetivo, es un solo espacio, son dos en uno. En esta relación el infante vive una invasión de su espacio, invasión que es necesaria y constitutiva del ser, donde hay una madre que se impone, que decide por el infante, muchas veces en disminución del placer, pero que tiene como objetivo la supervivencia biológica.

Esta invasión al espacio es considerada por Piera Auglanier, mencionada por Rojas (1998), como la primera manifestación de violencia que sufre el (la) infante, y la denomina como violencia primaria: "(...) radical y necesaria, que la psique del infans vivirá en el momento de su encuentro con la voz materna (...) 'designa lo que en el campo psíquico se impone desde el exterior a expensas de una primera violación de un espacio' (...)" (Rojas, 1998, p. 463). Cabe mencionar que esta violencia necesaria para la construcción del yo, se diferencia de la violencia secundaria, pues esta última, lejos de ser constitutiva, aniquila al yo, anula "(...) al otro como sujeto diferenciado, sumiéndolo en una pérdida de identidad y singularidad que señala el lugar de la angustia (...)" (Rojas, 1998, p. 463).

Por lo tanto, así como a través de la relación con la madre y otras personas, la (el) infante se nutre y alimenta, también va tomando los insumos significativos para la construcción de su subjetividad. Esta se inicia, entonces, a partir de las subjetividades de las demás personas alrededor, primeramente, a través de la madre con quien comparte el campo subjetivo y, 
posteriormente, por otros miembros de la familia, para ampliarse a otros individuos que conforman otros grupos primarios, como pequeños grupos de amigos y amigas, compañeros escolares o religiosos. La subjetividad responde según como se ha ido configurando el mundo interno del sujeto, en este caso, el del infante.

Es importante rescatar que la subjetividad es un constructo en continuo movimiento, no es acabado, ni finito, y está sujeto a cambios y variaciones que dependen de las situaciones de vida de los sujetos, por lo que sufre transformaciones que van acompañadas del enriquecimiento del mundo interno.

Bernard (1988b, p. 1) plantea que “(...) la identidad personal se produce a partir de la pertenencia a grupos primarios, especialmente la familia, y que se origina en la internalización de una serie de escenas significativas del sujeto, a partir de las cuales va construyendo su grupo interno (...)".

Esta escenas están construidas por personas, personajes, objetos parciales, objetos totales, roles y relaciones entre estos elementos. La identidad está basada en conjuntos de escenas internalizadas y, cuanto mayor sea la cantidad de escenas, más plástica y flexible es la identidad personal; al contrario, cuanto menos numerosas sean dichas escenas, más rígida e inamovible será la identidad y mayor trabajo será requerido para lograr un cambio.

Lo internalizado de la trama vincular familiar o escolar no es una réplica de la dinámica real del grupo primario: es una reconstrucción de lo significativo, que se convierte, posteriormente, en la realidad del sujeto.

Lo que se introyecta no es un objeto, sino una relación de objeto en la cual el sujeto está incluido. Tenemos entonces que la identidad del sujeto se produce a partir de la internalización de una sucesión de grupos en los cuales el sujeto ha participado, grupos que al principio son casi de dos personas, (madre-hijo) en la medida que el padre va entrando paulatinamente y que va pasando al momento de la castración simbólica pasan a ser grupos de tres elementos, un sujeto, su objeto y aquello que se interpone e interfiere entre ambos. (Bernard, 1988b, p. 8)

Ahora bien, si por parte del niño predomina una percepción indiscriminada con respecto a la relación con la madre (simbiótica) en la realidad externa, en el grupo interno predominará una estructura diádica, donde el guion internalizado va a estar impregnado por este modelo y donde predominará en sus vínculos la indiscriminación y fusión. Esta es una de las razones por las que la violencia primaria debe ir cediendo, para dar paso a la entrada de otros (terceros) que separen la fusión. 
Si, por el contrario, prevalece una percepción de separación de la diada madre-hijo, predominará, en sus vinculaciones posteriores, la tendencia a reconocer al otro sujeto como un ser diferenciado de sí mismo, a reconocerlo en su singularidad.

La forma en que es vivido este proceso de separación también es introyectado, porque se incorpora la forma de vinculación de los otros, entre ellos y consigo mismo. Tal como lo plantea Pichon-Rivière, mencionado por Bernard (s. f.), la internalización de la trama familiar es ecológica, porque incluye todo el alrededor, no solo las figuras parentales, hermanos, otros individuos significativos, sino también los objetos inanimados, el hábitat en su totalidad, no solo la dramática del contexto, sino además el ámbito físico en que se ha producido.

Ahora bien, si en el mejor de los casos los vínculos familiares, ambiente escolar y de comunidad (otros grupos en los que el individuo infante está inmerso) son fundamentados en el respeto a la individualidad y a la singularidad, estas formas de relación serán introyectadas y conformarán un referente para sus vinculaciones posteriores. Pero si, por el contrario, en la trama vincular en los grupos de pertenencia se viven situaciones traumáticas, como lo es la presencia de la violencia secundaria, las personas infantes sometidas a esto no solo interiorizarán el modo de relación, sino que pueden sufrir una conmoción que dificulta el procesamiento adecuado y favorece pactos de silencio.

Las familias violentas son grupos con creencias muy rígidas, grupos estereotipados donde la singularidad no es permitida y se intenta dominarla, controlarla y someterla. Tienden a vivir en aislamiento, para evitar que, a partir de las vinculaciones con otros grupos, sus miembros intenten o deseen modificar el contexto familiar. Este carácter endogámico y discurso incuestionable dificulta la incorporación de significaciones no violentas.

Algunos autores las comparan con regímenes totalitarios donde se instalan sistemas de vigilancia para lograr el sometimiento de sus integrantes. Por el contexto encerrador que viven sus miembros, estos vínculos son difíciles de romper, ya que la familia se promueve a sí misma como la poseedora de todas las respuestas y, a pesar del maltrato vivido, la familia brinda un lugar en esa dinámica particular, lugar desde donde se les reconoce, se les mira; es un lugar que da identidad, por lo que la separación es experimentada por sus integrantes como muerte a su identidad.

Tanto agresores como víctimas tienen "(...) déficit y excesos, sentidos y sin sentidos que operan transgeneracionalmente, actualizados con frecuencia a partir de condiciones violentas del presente que obstaculizan la resignificación de lo recibido y la apertura a lo nuevo (...)" (Rojas, 1998, p. 465). Se perpetúa su condición a través de la estereotipia o rigidez, tanto en los roles asumidos, como en los adjudicados a los demás y, en general, en la dinámica a jugar en la relación familiar.

La violencia es un poder extremo que conlleva a la destrucción de la subjetividad y puede manifestarse a través de: 
- Agresión física: Entendida como todo maltrato al cuerpo del otro/a.

- Abuso sexual: Todo acto en el que una persona en una relación de poder involucra a un menor en una actividad de contenido sexual (Rojas, 1998).

- Violencia discursiva: Incluye tanto el“(...) contenido semántico del discurso pero sobre todo la organización en lo implícito de lo enunciado (...) Se expresa en la palabra y el afecto, bajo diferentes modalidades discursivas (...)" (Rojas, 1998, p. 464). Este tipo de violencia, aunque parezca sutil, es tan devastadora como cualquier otra (Rojas, 1998).

- Desinvestidura de los vínculos: Cuando hay abandono en distintas modalidades y grados, cuando hay fallas en las ligaduras contenedoras y constitutivas del vínculo (Rojas, 1998).

Algunos mecanismos defensivos utilizados en el mantenimiento de la estereotipia son: la justificación, la renegación como forma de velamiento de las verdades y de distorsión que oscurece la violencia, y la desculpabilización del acto violento.

Según Rojas (1998), la posibilidad de un abordaje externo surge cuando se devela la situación. Generalmente quien favorece este develamiento es una persona extrafamiliar, que incluso puede ser la intervención judicial en los casos más severos.

\section{Aspectos socioculturales y transgeneracionales}

Lo social y lo cultural también son aspectos que están presentes en la formación del psiquismo y la subjetividad de los sujetos. Los significantes y significados socioculturales son introyectados en el mundo interno de los seres humanos.

Janine Puget, mencionada por Krakov y Pachuk (1998), plantea que la dimensión transubjetiva responde a un "(...) espacio de la mente, cuyas representaciones sociales están relacionas con ideología, religión, poder y pertenencia" (p. 447).

Por lo que cabe el cuestionamiento de cómo son trasmitidas algunas formas de violencia instituidas en el tiempo, como la violencia proveniente de la estructura social (pobreza, hambre, miseria, corrupción, explotación) y la violencia cultural, que utiliza el discurso del saber para legitimar diferentes formas de violencia (castigo físico, violencia en defensa propia, etc.).

Estas significaciones sociales son mediatizadas por las familias y los grupos (escolares, religiosos entre otros), con la transmisión de algunas y el rechazo de otras.

Los conjuntos intersubjetivos: familiares, de pareja o de grupos son trabajados por las significaciones sociales y por las creencias y mitos que ellos mismos instituyen (...). ( $\mathrm{L}^{\prime}$ Hoste, 1998, p. 212) 
(...) El discurso trasgeneracional se constituye como cadena de transmisión que involucra lo dicho, lo omitido y las condiciones de producción del discurso -lo cual refiere a la vehiculización implícita de la ideología (...). (Lamovsky, 1998, p. 442)

Esta red simbólica de significaciones permite que se instaure lo que Kaës (1995) denomina contrato narcisista, donde el sujeto introyecta los significantes socioculturales a cambio de redes identificatorias, y la pertenencia y lugar en la especie humana. Este contrato está presente desde el vínculo madre hijo y después está atravesando todos los grupos de partencia del sujeto.

La transmisión trasgeneracional“(...) se realiza en tensión entre un plano de reproducción y clausura, y otro de inestabilidad. Siempre se producen desacoples entre prácticas y enunciados que se propagan con fuerzas con posibilidad de instituir algo nuevo. Ambas vertientes nutren la forma de ser de los conjuntos intersubjetivos (...)" (L' Hoste, 1998, p. 212).

En familias violentas se tiende a reproducir y cerrar la posibilidad de inestabilizar la trama vincular establecida, imposibilitando la resignificación; se distorsiona, se descalifica o se invisibiliza la existencia de otra forma de vida y se opta por los patrones de violencia vividos generacionalmente.

En los ambientes educativos violentos también se tienden a reproducir los significantes socioculturales y a clausurar la posibilidad de cuestionamiento, tanto en los pequeños grupos de infantes, donde se exige la homogenización y el discurso del docente se vuelve incuestionable y no se reconoce a los/as otros/as como sujetos diferentes entre sí, negando la singularidad de pensamiento y palabra, como a un nivel estructural organizativo, donde la violencia y abuso de poder están instituidos bajo el discurso del saber y pasan desapercibidos por los diferentes actores educativos.

\section{Dimensión vincular, los vínculos posteriores}

Las relaciones que estos infantes establecerán con los otros estarán ligadas directamente con las escenas introyectadas en sus grupos internos. "(...) Los vínculos entre el yo y los objetos internos marcan de una manera muy fuerte el vínculo externo (...) lo que el sujeto coloca afuera, en el mundo exterior o en la sociedad, es la pauta de conducta de los vínculos internos con sus objetos internos (...)" (Pichon-Rivière, 1985, pp. 51-52). Los vínculos que establecerán funcionarán para estos sujetos como pantallas receptoras de una relación vincular anteriormente introyectada; la vinculación que tiene en su mundo interno actuará en estas nuevas relaciones.

Bernard (1988a) considera que en toda vinculación hay dos polos: uno adaptativo que da cuenta de la posibilidad de ver al otro como sujeto singular, diferente de los introyectados en su psiquismo y, por lo tanto, favorece la posibilidad de no reproducir una forma de vinculación 
anterior. Y otro polo imaginario, que se observa cuando la persona no es capaz de reconocer al otro/a con quien se relaciona como un ser diferente del introyectado anteriormente, y proyecta sobre él/ella la sombra de una dinámica vincular interna.

Todo vínculo dispara este aspecto imaginario, pero en las personas con historia de violencia, este aspecto suele tener mayor fuerza, por lo que tienden a repetir la dinámica violenta vivida.

La posibilidad de un mayor acercamiento al polo adaptativo dependerá de la discriminación vivida en los primeros vínculos internalizados, pues a más discriminación interna, más discriminación en los vínculos externos y más posibilidad de resignificación y de modificaciones de las relaciones vinculares.

Si en el vínculo predomina el polo imaginario, la proyección de la estructura interna sobre la estructura de roles del contexto intersubjetivo será mucho mayor y, por consiguiente, habrá más posibilidad de emergencia del conflicto. Esto en su extremo produce violencia, ya que no se reconocería al otro como sujeto de deseo.

Lo anterior lleva a concluir que la presencia de la violencia en la construcción de la identidad teñirá de violencia la visión de mundo y la subjetividad de quienes la vivieron, condenándolos a la repetición y perpetuación de esta forma de vinculación.

Así mismo, cabe considerar que la posibilidad de cambio dependerá en primer lugar del deseo, que de manifestarse, significa que la persona tiene la capacidad de percibir la existencia de formas de relación diferentes de las que posee (más cercanas al polo adaptativo) (Bernard, 1988a), lo cual permitiría, trabajando en ello, poder introyectarlas y enriquecer su grupo interno.

Esta modificación facilitaría al sujeto la re-significación de su historia y, por ende, su subjetividad. Esto no es tarea sencilla ni rápida; sin embargo, no imposible. Con deseo, disposición, esfuerzo y un buen acompañamiento individual o grupal, se puede lograr.

\section{Referencias}

Bernard, M. (1988a). Grupo interno transferencia horizontal y vertical (Maestría en estudios en Psicología Grupal, clase 6). En UCl, Antología: Desarrollo a través de la cooperación (pp. 1-23). Argentina: Instituto de psicoanálisis de las configuraciones vinculares.

Bernard, M. (1988b). Interacción entre grupo interno y grupo externo (Maestría en estudios en psicología Grupal, clase 9). En UCl, Antología: Desarrollo a través de la cooperación (pp. 1-9). Argentina: Instituto de psicoanálisis de las configuraciones vinculares.

Bernard, M. (s. f.). Historia del concepto de grupo interno. Enfoque sociológico, psicosocial y psicoanalítico (Maestría en estudios en Psicología Grupal, Clase 5). En UCl, Antología: Desarrollo a través de la cooperación (pp. 1-14). Argentina: Instituto de Psicoanálisis de las Configuraciones Vinculares. 
URL: http://www.una.ac.cr/educare

Kaës, R. (1995). El inconsciente y las alianzas inconscientes. Investigaciones para una metapsicología de los conjuntos intersubjetivos (Cap. 7). En. R. Käes (Aut.), El grupo y el sujeto del grupo: Elementos para una teoría psicoanalítica del grupo (pp. 287-338). Buenos Aires: Amorrortu.

Krakov, H y Pachuc, C. (1998). Tres espacios psíquicos. En C. Pachuk y Friedler, R. (Coords.). Diccionario de psicoanálisis de las configuraciones vinculares (pp. 445-450). Buenos Aires: Ediciones del Candil.

L' Hoste, M. L. (1998). Imaginario social. En C. Pachuk y Friedler, R. (Coords.). Diccionario de psicoanálisis de las configuraciones vinculares (pp. 207-215). Buenos Aires: Ediciones del Candil.

Lamovsky, C. (1998). Transmisión transgeneracional de las significaciones. En C. Pachuk y Friedler, R. (Coords.), Diccionario de psicoanálisis de las configuraciones vinculares (pp. 441444). Argentina: Editorial Del Candil.

Pichon-Rivière, E. (1985). Vínculo, comunicación y aprendizaje (Cap. 3). En E. Pichon-Rivière (Aut.). Teoría del vínculo (pp. 47-56). Buenos Aires: Nueva Visión. Recuperado de http:// es.scribd.com/doc/89114552/Libro-Teoria-Del-Vinculo

Rojas, C. (1998). Violencia familiar. En C. Pachuk y Friedler, R. (Coords.), Diccionario de psicoanálisis de las configuraciones vinculares (pp. 463-468). Argentina: Editorial Del Candil.

\section{Cómo citar este artículo, según APA:}

Amador, M. (2012). Hacia una lectura de la violencia en la formación de la subjetividad Revista Electrónica Educare, 16 (Especial), 31-38. Consultado de http://www.revistas.una.ac.cr/index.php/EDUCARE/issue/view/418

Nota: Para citar este artículo en otros sistemas puede consultar el hipervínculo "Como citar el artículo" en la barra derecha de nuestro sitio web: http://www.revistas.una.ac.cr/index.php/ EDUCARE/index 